\title{
Atom Probe Tomography of Interfaces at the Near-Lattice Level
}

\author{
P. J. Felfer, S. P. Ringer and J. M. Cairney
}

The Australian Centre for Microscopy \& Microanalysis, The University of Sydney, NSW 2006, Sydney, Australia.

The development of a reproducible site-specific specimen preparation method has enabled the routine investigation of grain boundaries and hetero-phase interfaces in engineering materials and model alloys using atom probe tomography (APT). Utilizing the high spatial resolution when interfaces are positioned perpendicular to the measurement direction and the high chemical sensitivity of APT, the distribution of elements around grain boundaries has been investigated on a near lattice level in a novel strip-cast steel, modifications of the Ni-based superalloy A718, two different $\mathrm{Al}$ alloys, high purity $\mathrm{Fe}$ and $\mathrm{Fe}$ with $\mathrm{C}$ additions.

The spatial resolving power of the atom probe is shown to be preserved when grain boundaries are measured close to perpendicular to the specimen axis [1]. While lattice resolution at a grain boundary has been achieved for pure Fe [2], solutes are detected at significantly different positions to their original ones in the bulk material. It is widely accepted that lateral displacement of the solute atoms can be attributed to field-induced diffusion across the tip surface, but here we also suggest that incorrect positioning of solute atoms in the $\mathrm{z}$ direction is the result of preferential retention of these species on the atom probe tip. Preferentially-retained atoms are detected later in the evaporation sequence and therefore be assigned an incorrect depth coordinate (fig.1). Depending on the matrixsolute combination, solutes atoms may be retained on the tip surface while several atomic layers of material are removed. This effect is especially prominent for high-field species, but species with similar evaporation fields to the matrix may also experience significant preferential retention. This effect makes analysis of the grain boundary region at the crystal lattice scale challenging, since the positions of individual atoms will not be correctly reproduced and interfacial concentrations will be underestimated.

However, the retention has a statistical component, which allows for the derivation of a simple decay law describing the effect. This decay law can be interpreted as a species-specific point spread function, describing the relative probability of evaporation of the solute compared to the matrix. Although the individual atomic positions of the solutes cannot be recovered, the concentration profile can be therefore rectified by deconvolution. This is demonstrated for $\mathrm{Cu}$ at an $\mathrm{Al}$ grain boundary (atom map is displayed in fig. 2), where after deconvolution the non-equilibrium segregation caused by water-quenching becomes apparent.

Besides the identification of different chemical elements, the atom probe is able to distinguish between different isotopes of a given chemical element through its time-of-flight principle. This makes it ideal for self-diffusion experiments at lattice defects such as grain boundaries and dislocations. An experiment will be presented that uses the diffusion of stable tracer isotopes at different types of grain boundaries in order to reveal their individual self-diffusion coefficients and energies.

References

[1] D. Blavette et al., Acta Mat., 44 (1996) 4995.

[2] P. Felfer et al., Ultramicroscopy, In press. 
[3] This work was funded by the Australian Research Council (ARC). The authors are grateful for scientific and technical input and support from the Australian Microscopy \& Microanalysis Research Facility (AMMRF) node at the University of Sydney.

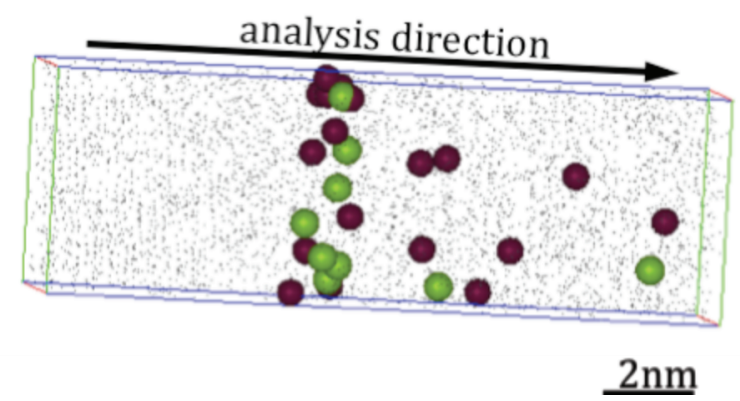

FIG. 1. Atom probe reconstruction of a grain boundary in Fe containing C. While the lattice positions of the matrix are largely preserved across the boundary, $\mathrm{C}$ atoms are found mostly below the boundary due to preferential retention (brown: $\mathrm{C}$, green: $\mathrm{C}_{2}$ molecular ions).

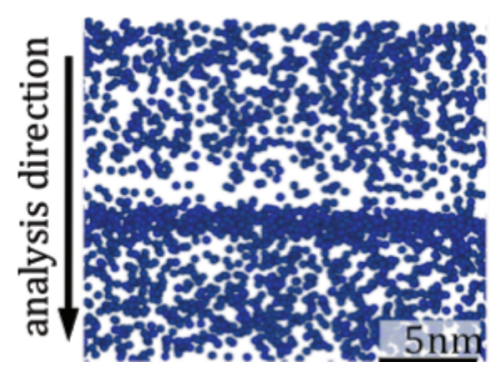

FIG. 2. Atom map of $\mathrm{Cu}$ around a grain boundary in $\mathrm{Al}$. A depletion of $\mathrm{Cu}$ with respect to the bulk concentration is evident around the grain boundary only above the segregated area. 